\title{
Composite of Tin and Silicon with Nanostructure as High Performance Lithium-Ion Battery Anode
}

Chao Wang, Xiufang Bian*, Yinghui Yang, Chao Yuan, Junzhang Wang, Mengchun Yu, Rongzhang Guan, Dujiang Lu

Key Laboratory for Liquid-Solid Structural Evolution and Processing of Materials, Ministry of Education, Shandong University, Jinan 250061, China

*E-mail: xfbian@sdu.edu.cn

doi: $10.20964 / 2020.04 .34$

Received: 7 December 2019 / Accepted: 13 February 2020 / Published: 10 March 2020

Composite of nanobranches-Si and nanospheres-Sn (denoted as $\mathrm{Sn}^{\#} \mathrm{nb}-\mathrm{Si}$ ) with an appearance of "fruitbranch" is successfully synthesized by two-step method at room-temperature. This two-step method, i.e. dealloying followed by depositing, constructs an intact network of nanobranches-Si (nb-Si) with a harmonious depositing of nanospheres-Sn. The structural design at the nanoscale level buffers the expansion during lithiation/delithiation process, and the depositing of nanospheres-Sn improves the rate of $\mathrm{Li}^{+}$transfer (an increase of $145 \%$ compared to pure $\mathrm{nb}-\mathrm{Si}$ ), remedying inherent poor conductivity of $\mathrm{Si}$ and achieving enhanced electrochemical performance. As a result, $\mathrm{Sn}^{\#} \mathrm{nb}$-Si exhibits a reversible capacity of $1525.9 \mathrm{~mA} \mathrm{~h} \mathrm{~g}^{-1}$ at $100 \mathrm{~mA} \mathrm{~g}^{-1}$ after 55 cycles with decent rate performance. Furthermore, $\mathrm{Sn}^{\#} \mathrm{nb}$-Si deepens research on the composite of $\mathrm{Si}$ and $\mathrm{Sn}$, while dealloying followed by depositing, which is generally facile and uncomplicated, offers a novel guideline to cultivate structural design and paves the way for the synthesis of advanced energy storage materials.

Keywords: LIBs, Si/Sn, anode, composite, dealloying, depositing

\section{$\underline{\text { FULL TEXT }}$}

(C) 2020 The Authors. Published by ESG (www.electrochemsci.org). This article is an open access article distributed under the terms and conditions of the Creative Commons Attribution license (http://creativecommons.org/licenses/by/4.0/). 